Diversidad cultural e implicaciones en la enseñanza de las ciencias: reflexiones y avances

Adela Molina Andrade Carmen Alicia Martínez Rivera Carlos Javier Mosquera Suárez Lyda Mojica Ríos 


\section{Resumen}

El presente artículo se configuró a partir de la versión del marco teórico y los antecedentes del proyecto de investigación "Concepciones de los profesores de ciencias sobre la diversidad cultural y sus implicaciones en la enseñanza" ${ }^{1}$. Se inicia con una idea de diversidad cultural, apoyada en Mauss, Douglas y Bruner. Luego se argumenta cómo el problema de la diversidad implica emergencias y complejidades que incluyen lo político, la configuración de la identidad y la memoria cultural, que actúan como un mecanismo de recuerdo y olvido. En esta dialéctica, se describen algunos trazos de nuestra propia constitución cultural.

Con este marco se caracterizan posibles relaciones entre diversidad cultural y educación. Así, se pueden destacar varias reflexiones, entendidas como un abreboca para iniciar la discusión en torno a lo educativo y en relación con lo diverso, apareciendo entonces, notas para comprender la homogeneización en la educación y las relaciones entre diversidad cultural, educación y enseñanza de las ciencias. La polaridad descrita se discute en el marco de la educación en ciencias como campo de investigación, ya que es tal vez en el que los debates sobre homogenización y la diversidad se manifiestan con gran "efervescencia", dado que la ciencia encarna uno de los pilares más importantes de la modernidad.

\section{Palabras clave}

Diversidad cultural, educación en ciencias, concepciones de los profesores, modernidad, epistemología y cultura.

\footnotetext{
${ }^{1}$ Proyecto ejecutado por el Centro de Investigaciones y Desarrollo Científico de la Universidad Distrital Francisco José de Caldas (CIDC-UD) con cofinanciación de Colciencias. El equipo está constituido por Adela Molina, Carmen Alicia Martínez, Lyda Mojica, Mauricio Lizarralde, Carlos Javier Mosquera, Graciela Utges, Rosa Inés Pedreros, Duván Reyes y María Cristina Cifuentes
}

\section{Abstract}

This paper derived from a version of the theoretical framework and background of the research project "Conceptions of science teachers about cultural diversity and its implications for teaching". It begins with an idea of cultural diversity, supported by Mauss, Douglas and Bruner. We argued that cultural diversity and complexities involve emergencies that include politics, shaping the identity and cultural memory. These, in turn, act as a mechanism of memory and forgetfulness. In this dialectic we can find some traces of our own cultural origin. With this framework, it is possible to make links between cultural diversity and education. Thus, several ideas can be highlighted and understood as an introduction to thinking about education and its relation to diversity. Mentions are made around the topic of homogenization in education and its relationships with cultural diversity and education. The polarity described is discussed in the context of science education since it is perhaps one of the fields in which the discussions on harmonization and diversity is manifested with greater "ferment" and science is considered one of the pillars of Modernity.

\section{Key words}

Cultural diversity, science education, teacher's conceptions, modernity, epistemology and culture. 


\title{
Diversidad cultural e implicaciones en la enseñanza de las ciencias: reflexiones y avances ${ }^{1}$
}

\author{
Adela Molina Andrade ${ }^{2}$ \\ Carmen Alicia Martínez Rivera ${ }^{3}$ \\ Carlos Javier Mosquera Suárez ${ }^{4}$ \\ Lyda Mojica Ríos ${ }^{5}$
}

Producimos cultura de igual manera que la remolacha,
en régimen de monocultivo.

Lévi-Strauss

El campo de la educación en ciencias tampoco escapa de las discusiones sobre la diversidad cultural, este debate se explicita en consideraciones sobre el multiculturalismo y, fundamentalmente, en sus implicaciones epistemológicas, ontológicas y morales; otras reflexiones se derivan del análisis de la educación científica en contextos culturales particulares. De acuerdo con lo anterior, es necesario acudir a conceptos y desarrollos de otros campos, este es el caso de algunos antecedentes para perfilar una idea de diversidad cultural. En el caso de Velho (1978, p. 6), se desarrolla el concepto de modalidad de Mauss, y nos muestra importantes perspectivas de aplicación para la educación; “[...] la humanización del hombre siempre se realiza a partir de

\footnotetext{
${ }^{1}$ Texto recibido en 3 de abril, evaluado en el 16 y el 23 dejunio 16 y arbitrado en el 30 de junio de 2009 2 Doctora en Educación, Área Didáctica de las Ciencias Naturales, Universidad de San Pablo, Brasil. Profesora Facultad de ciencias y educación de la Universidad Distrital Francisco José de Caldas, en el Doctorado Interinstitucional en Educación. adela@udistrital.edu.co

${ }^{3}$ Doctora en Educación, Programa Didáctica de las Ciencias Experimentales y Sociales, Universidad de Sevilla, España. Profesora Facultad de Ciencias y Educación de la Universidad Distrital Francisco José de Caldas, en el Doctorado Interinstitucional en Educación. camartinezr@udistrital.edu.co

${ }^{4}$ Doctor en Didáctica de las Ciencias Experimentales, Universidad de Valencia, España. Profesor Facultad de Ciencias y Educación de la Universidad Distrital Francisco José de Caldas, en el Doctorado Interinstitucional en Educación y en la Licenciatura en Química. cmosquera@udistrital.edu.co

${ }^{5}$ Magíster en Educación, Pontificia Universidad Javeriana. Profesora Facultad de Ciencias y Educación en la Licenciatura en Pedagogía Infantil, Universidad Distrital Francisco José de Caldas. Imojica@udistrital.edu.co
} 
un modo de vida particular [...]", esto es, el hombre posee percepciones, lenguajes y formas de pensar moldeados por las culturas. Este concepto de modalidad significó, para la antropología, la internalización de la diversidad cultural como una "realidad" a ser investigada (Velho, 1978).

De esta forma, el ámbito cultural no comporta una determinación universal; como dice Mauss "[...] el dominio de la cultura es la modalidad [...] la selección cultural, y en particular, la relacionada con las capacidades biológicas a ser desarrolladas o inhibidas, todo esto podría ser de otro modo" (Velho, 1978, p. 6.). "Es así como la humanización del hombre siempre se realiza a partir de un modo de vida particular [...] el hombre no se realiza a través de una humanidad abstracta (LéviStrauss, 1973, citado en Velho, 1978, p. 6.)". En este sentido:

Mauss describió una gran variedad de técnicas corporales, pero no como resultado exclusivo de una actividad individual, sino fundamentalmente, como resultado de su educación, de toda la sociedad de la cual ellos hacen parte, en el lugar que él ocupa (Mauss, 1974, p. 218, citado en Gomes, 2003, p. 80).

Con el anterior enfoque, Mary Douglas (1998) investigó el vínculo entre estructuras sociales, modos de pensamiento y su expresión en sus comportamientos. De igual modo, Bruner (1990, p. 47) señala que los requerimientos de los estilos de vida dan sentido y explican el funcionamiento de la mente:
[...] Para comprender al hombre; es necesario, primero, comprender cómo sus experiencias y actos están moldeados por estados intencionales y segundo, que la forma de dichos estados intencionales, sólo puede plasmarse mediante la participación en los sistemas simbólicos de la cultura. Es la cultura la que moldea la vida y las mentes humanas, la que le confiere significado a la acción situando sus estados intencionales subyacentes en un sistema interpretativo.

Aquí se encuentra el valor de lo educativo, pues los sistemas educativos, desde el punto de vista cultural, se entienden como procesos de preservación y dinamización de las culturas. Para Forquin (1993), dicha dinamización se realiza mediante procesos de selección cultural; en una cultura, tales elecciones poseen una doble importancia. Por medio de ella se realiza una reelaboración de los contenidos que son transmitidos a las nuevas generaciones. Pero no es transmitida como un repertorio simbólico unitario, dicha transmisión está sujeta a los azares de las relaciones simbólicas y a los conflictos de interpretación.

La diversidad cultural implica aspectos políticos, jurídicos, epistémicos, ontológicos y axiológicos muy difíciles de separar. A partir de Subercaseux (2005, pp. 45-50), vemos que su comprensión de lo político permitiría una forma de entender lo nacional que evita la polaridad iluminismo-romanticismo de la perspectiva occidental: 
[...] En tiempos globalizados la diversidad cultural constituye una oportunidad para reformular y revitalizar el imaginario de la nación, articulando lo político y lo cultural. Se trata de construir una nación que no sea excluyente, que no margina, una nación con nuevos aires, en que el nosotros conviva y respete al otro, tanto al interior de la nación como a nivel de países y continentes.

En el contexto de esta crítica, es posible comprender a Canen y De Oliveira, (2002) y Molina (2000, 2005), quienes se preguntan sobre la constitución de la identidad y el reconocimiento del otro en el campo educativo. Así, es clara la alusión de Carlos Gutiérrez (1997, p. 72), que integra la dinámica entre alteridad-identidad y política:

En general las legislaciones republicanas en América Latina se esforzaron por llenar el espacio más o menos abstracto del estado con una identidad colectiva, a partir de una uniformización nacionalista [...] pese a tal heterogeneidad, diversidad y fragmentación cultural [...] en el hispanismo -ratificado en la constitución colombiana de 1886- que apuntalaba las instituciones de educación y de adoctrinamiento para grabar en la conciencia y en la subconciencia de los nacionales la superioridad de una raza, suponiendo que nuestros pueblos sólo llegarían a la democracia si llegaban a ser idénticos, dándose así la paradoja de que muchos hayan tenido que renunciar a su identidad cultural para adquirir derechos ciudadanos.

Sin embargo, podemos comprender a partir de Pires Ferreira (1995), que este intento de ingreso a la modernidad de nuestro país fue paradójico y contradictorio. Por una parte, implicó dominación y exclusión de la diversidad, en el mejor de los casos, la buena intención de hacer a todos iguales, dado que "[...] el olvido es un mecanismo utilizado por una institución hegemónica, que busca excluir de la tradición los elementos indeseables de la memoria colectiva” (p. 117). Por otra parte, la exclusión no pudo ser total. Guillen (1996, p. 53) muestra que la experiencia de los colonizadores y su interés inmediato enseñaron que la sujeción indígena y su "enseñanza" solamente eran viables, utilizando los valores y las actitudes ancestrales de los grupos precolombinos. Como anota Pires Ferreira (p. 117), "es interesante ver cómo ahí el olvido se puede transformar en un mecanismo de memoria, pues una cultura se concibe a sí misma como existente cuando se identifica con las normas constantes de la propia memoria".

Para una mayor comprensión de tal polaridad, es necesario reconocer que la dispersión y fragmentación de la sociedad colombiana (Tovar, 1993) implica definir políticas, prácticas y actitudes de reconocimiento, adecuadas a nuestra condición, para hacer de dicha diversidad una posibilidad viable en nuestra sociedad. Como afirman Hederich y Camargo (1999: p. 99) es "[...] el desconocimiento, por parte de las clases en el poder, de la naturaleza multiétnica regional colombiana lo que po- 
dría estar en la base de los profundos desequilibrios y conflictos que aquejan nuestro país. En el respeto, la consciencia y el aprovechamiento de nuestras diferencias podrían estar las respuestas [...]". Pero no se trata de asumir una polaridad estéril; parafraseando a Santos (2003, p. 10. citado por Candau y Nehme, 2003, p. 474), es necesario vincular la igualdad y diferencia, entendiendo que aquello que se opone a la igualdad es la desigualdad y a la diferencia es la homogenización: "Las personas y los grupos sociales tienen el derecho a ser iguales cuando la diferencia los hace inferiores y el derecho de ser diferentes, cuando la igualdad los descaracteriza".

\section{Notas para comprender la homogenización en la educación}

En el anterior contexto, y para comprender la discusión sobre cómo posturas homogenizadoras del ciudadano, (aquel que participa en la vida política de su país) tienen su correlato en el discurso sobre la constitución del sujeto y de lo educativo, en términos cognitivos y epistemológicos, el universalismo representa tal perspectiva de estandarización y se fundamenta en una visión iluminista. Su contrapunto, el romanticismo, se puede entender como una posibilidad para asumir el reconocimiento de lo diverso y heterogéneo en cuanto a lo cognitivo y conceptual; tal denominación es tomada de Shweder (1991), cuidando de no caer en la polaridad iluminismo-romanticismo, como lo advierte Subercaseux (2005).

La argumentación de Shweder (1991) sobre el iluminismo y sus implicaciones, en la concepción de sujeto e idea de cognición que de ella se deriva, debate la concepción de cultura de Tylor y Frazer, pues en esta perspectiva la mente del hombre es intencionalmente científica y racional.

Por otra parte, los promotores de esta idea también asumen que existen pueblos que no razonan y valoran las evidencias correctamente; ellos son pueblos primitivos, Tylor y Frazer, promotores de la distinción entre “modernos" y "primitivos”, afirman que "los primitivos respetan la razón y la evidencia, pero fracasan en la aplicación de los cánones apropiados de lógica, estadística y ciencia experimental" (Shweder, 1991, p. 82). Cabe recordar que desde la reciente investigación en didáctica de las ciencias (Pozo y Gómez Crespo, 1998; Pozo, 2003) se insiste en superar la idea habitual de jerarquizaciones entre formas de conocimiento diferentes (como el conocimiento cotidiano y el conocimiento escolar, por ejemplo). Otra cosa es que operen lógicamente diferente, pero en últimas son conocimientos importantes que surgen en las culturas.

Volviendo a la discusión sobre homogenización, ésta encuentra eco y se instaura en nuestra propia visión del otro (que conoce, piensa, siente) y por tanto de nosotros mismos, con importantes implicaciones en la educación; así, los argumentos de Frazer y Tylor constituyen un ideal: ser racionales, hacer inferencias y explicar el mundo para llegar a ser "modernos” y así abandonar la condición de "primitivos". 
Se asume la misma estrategia iluminista, que supone que todos los hombres se inclinan ante la razón y la evidencia, y que ellas (las estrategias) son las mismas para todos, lo que implica que los sujetos evalúan los sucesos, las contingencias, los hechos con los mismos criterios. Unidad y uniformidad se ajustan, la primera aboga únicamente por el respeto de la autoridad de la razón y la evidencia, la segunda considera que las conclusiones fundamentales de cómo vivir y en qué creer son uniformes para todos, aspectos también establecidos mediante la razón y la evidencia.

La pregunta sobre si el conocimiento válido, que se establece mediante la razón y la evidencia, es el mismo para todos, conduce a dos formas de respuesta para los iluministas: la respuesta universalista y la evolutiva (Shweder, 1991, p. 83). Los primeros afirman que los principios universalmente válidos, de la razón y la evidencia, son obvios, y por tanto asequibles y “[...] comprensibles por los niños". Los segundos, los evolucionistas:

[...] Niegan típicamente que los dictámenes universalmente válidos de la razón y de la evidencia estén disponibles por igual para todas las personas y todos los pueblos [...] Tylor, Frazer y más recientemente Piaget afirman que los estándares normativos (por ejemplo la lógica) -por referencia a la cual una persona o pueblo juzga adecuado o no al pensamiento y la acción- experimentan desarrollo [...] Según esta versión aunque [...] todos los pueblos posean normas para evaluar el pensamiento y la acción [...] pero el conocimiento de estándares adecuados [...] el conocimiento de esas normas merecedoras de respeto universal, es alcanzado sólo por unas pocas culturas (las civilizadas).

Continuando con Shweder (1991, p. 101), con los evolucionistas encontramos, en la idea de progreso, un incesante esfuerzo por lograr mejores adaptaciones:

De acuerdo con Piaget, la mente del niño crece en términos de etapas, yendo del pensamiento temprano concreto, indiferenciado y ligado con el contexto al pensamiento maduro abstracto, diferenciado y generalizado. La mente inmadura se aferra al contenido de superficie; la mente madura libre de contexto abstrae la estructura profunda.

Con lo anterior se agrega un nuevo criterio clasificatorio y discriminatorio; no solo existen mentes y concepciones "primitivas" y "modernas", sino que también existen pensamientos, razonamientos más evolucionados y mejor adaptados, unos más que otros, y la condición de los razonamientos de los niños o de aquellos que se le parecen poseen, por definición, una condición de "inmadurez". Son pues solidarios históricamente con esta idea de homogenización (presente en nuestro imaginario) los ideales inspirados en el pensamiento "iluminista" de lograr un pensamiento racional que resuelve problemas correctamente, o sea, como lo haría un científico 
(en sus modalidades universal y evolutiva); así uno (la política por la homogeneidad) y otro (el iluminismo) tengan significados y orígenes diferentes. En el primer caso, se trataba de lograr una identidad (por ejemplo, lograr ser como aquellos que nos colonizaron). En el segundo caso, se trataba de la concreción del proyecto de la "modernidad", de desarrollarlo en el campo intelectual.

\section{Una primera comprensión de la diversidad en el contexto de la educación}

En el caso de la educación, la compresión de la diversidad cultural implica compromisos y aperturas por parte de la escuela, los profesores y los demás actores que participan en ésta. Para configurar horizontes hacia tales compromisos y aperturas, se insistirá en dos aspectos, 1 . Uno que se refiere a la actitud orientadora de una acción abierta a la diferencia y lo heterogéneo, 2. La discusión sobre un enfoque que asuma la condición de lo diverso y heterogéneo en relación con lo cognitivo, las concepciones y cosmovisiones del sujeto.

Es posible asumir los puntos de vista de Langon (1999) y Llyod (1995), y sus tesis de una “ontología histórica, como referencia a la hora del análisis de una actitud orientadora de la acción hacia la apertura; éstos han sido sintetizados por Molina (2005, p. 141) e implican la necesidad de posturas que asuman que el diálogo entre diferentes sujetos y culturas requiere condiciones horizontales; la comprensión de tales encuentros “[...] sería el campo de una ontogénesis histórica y [...] se asume que dicha ontología no parte de cero”. Y como anota Langon (1999, p. 21), los sujetos que interactúan deben ser pensados desde dicha interacción y no desde los individuos abstractos; se asume entonces que cuando nos encontramos con alguien que no comprendemos, nos invade la necesidad de la apertura y la superación del aislamiento, así los espacios de encuentro se generan de la necesidad del otro y de lo otro.

Los encuentros permiten a cada sujeto actualizar su propia cultura, pero éstos son inéditos y una vez abiertos quedan “preñados de posibilidades” (Langon, 1999, p. 21). En los términos de Boaventura (1989), Molina (2002), Toulmin (1977) o D’Ambrosio (1998), entre otros, los etnocentrismos (racionalista, cultural, etc.) no permiten la apertura y el diálogo intercultural.

La referencia de Toledo (1998), cuando propone su idea de una etnografía cognitiva de la epistemología y da cuenta de los aportes de Feyerabend a su perspectiva, ilustra respecto de la crítica del etnocentrismo occidental y en particular, el inspirado y ejercido desde perspectivas racionalistas del conocimiento científico; así, anota: "Sería una brutalidad extrema interpretar nuestros propios logros insignificantes como si fueran universalmente obligatorios o como si tuvieran que ser tomados en consideración por todo el mundo y con toda seriedad" (Feyerabend, 1993, citado en Toledo, 1998, p. 32).

En tal contexto es importante la discusión sobre la diversidad de cogniciones, concepciones y cosmovisiones del sujeto, en la reflexión propuesta por Shweder 
(1991) sobre el iluminismo y el romanticismo. Complementa un marco comprensivo sobre el otro, su pensamiento y la revalorización positiva acerca de la condición diversa y heterogénea de nuestra cultura. En su análisis, al colocar el iluminismo en contrapunto del romanticismo, ayuda a explicitar aspectos no considerados, aunque tal reflexión conlleve polarizaciones.

Las ideas y prácticas no siempre se fundamentan en la lógica y en la ciencia empírica; existen casos en lo cuales los cánones de lo racional, como la validez, están fuera de lugar. Según Shweder, para los iluministas, quienes no proceden de acuerdo con tales cánones, son irracionales; así, la permanencia latente de una herencia fundamentada en una ideología de corte universalista, se constituye en criterios excluyentes de lo diverso y lo diferente. Como anota Escobar (2003, p. 56):

La modernidad introduce un orden basado en los constructos de la razón, el individuo, el conocimiento experto y los mecanismos administrativos ligados al Estado. Orden y razón son vistos como el fundamento para la igualdad y la libertad, posibilitando así el lenguaje de los derechos.

Los resultados de las investigaciones en antropología cognitiva (citados por Shweder) amplían una visión crítica; se observa que la mayoría de las veces las personas actúan con una mentalidad "primitiva", como anota Shweder: "No somos buenos aplicando la ciencia”. Una postura más relativista permitiría comprender que este dominio humano de lo racional debería considerarse como una de las posibilidades de la acción del pensamiento. La superación de la disyuntiva racional-irracional nos permite avizorar el dominio de lo no-racional; parafraseando a Shweder, ese dominio se refiere a la:

[...] Cultura, un particionamiento del mundo no racional, extralógico, arbitrario, que es enmarcada, referida, actuada y aún rotulada y que se transmite de una generación a la siguiente [...] Puedes comer conejos y ovejas, pero no perros o caballos. ¿Pueden la lógica y la ciencia habernos dicho eso? (Shweder, 1991, p. 97).

Este dominio de lo no-lógico, del mundo tal como se presenta, no está ahí para ser descubierto, es caleidoscópico, es organizado por nuestras mentes, para lo cual dependemos de los marcos de referencia que le dan sentido a las afirmaciones sobre el mundo y la vida, es lo que las hace inteligibles. En este sentido, el estudio de "[...] la ontogenia de las ideas en los niños [...] conduce al romántico a preocuparse por la adquisición de las ideas como comunicación tácita" (Shweder, 1991, p. 101). Este proceso se refiere a la socialización de los significados culturales, que pasan a ser parte del contenido de los marcos de referencia (valores, presupuesto, esquemas) de las generaciones siguientes, y que se constituyen en materia prima para toda construcción conceptual. 
Uno de los aportes de la nueva filosofía de la ciencia para la renovación de la enseñanza de las ciencias y fundamentalmente para el enfoque constructivista, se refiere a que el mundo no es como es, sino como nuestros conocimientos, creencias y perspectivas lo ven. Así, la enseñanza de las ciencias no debería orientarse a aprender a leer un libro ya escrito. Para Stanley y Brickhouse (2001), esta perspectiva epistemológica realista es sostenida por posturas universalistas, y en el campo de la educación en ciencias -que contradicen perspectivas constructivistas y multiculturales- sostienen que la ciencia occidental moderna se trata de una "[...] actividad intelectual cuyo objetivo fundamental no es afectado por la nacionalidad, clase, raza u otro" (Matthews, 1994, p. 182, citado por Stanley y Brickhouse, 2001, p. 37.)

\section{Entre la homogenización y la diversidad: debates en la enseñanza de las ciencias}

Para iniciar, es necesario plantear varias ideas que serán útiles para referirse a la diversidad cultural: multiculturalismo tradicional, multiculturalismo crítico e interculturalidad. En primer término, encontramos a Tyler (1993), quien busca los antecedentes para una política del reconocimiento en el ámbito de lo multicultural, justificada en aquello que debe ser transmitido y considerado por su valor estético o intelectual mediante la escolaridad, sin importar el origen cultural de cada sujeto. En el caso de López (2000), se puede entender esta perspectiva de multiculturalismo tradicional, en el ámbito anglosajón, como coexistencia de diversas culturas y lenguas en un mismo espacio.

Este es uno de los aspectos que caracterizan el actual mundo globalizado, debido, entre otros aspectos, a la creciente movilidad e intercomunicación características de nuestro tiempo, situación que ha llevado a la coexistencia, por una parte, de perspectivas homogenizadoras y, por otra, de una gran heterogeneidad que implica la presencia de diferentes culturas; desde esta perspectiva, Sabariego (2002, citado en López, 2000) anota que el término que se utiliza en el ámbito anglosajón se refiere a "[...] todas las diferencias de perspectiva moral o identidad personal basadas en la pertenencia a un grupo u otro", haciendo prevalecer la diferencia y dando cabida a cuantos modelos se plantea su explicación, sea ésta de la posición ideológica que sea. De este modo, podemos encontrar con el término "multicultural” propuestas, tanto de corte asimilacionista, como de corte socio-crítico.

Para los multiculturalistas críticos (Giroux, 1997), ello implica intercambios en las fronteras de la culturas y también un compromiso verdadero con las diferencias; García (2004) fundamenta el concepto de cultura desde las diferencias, los contrastes y las comparaciones antes que en las propiedades de los individuos y grupos; la entiende como un recurso heurístico para hablar de la variedad. De acuerdo con Moreira (2002), se propone el multiculturalismo crítico como respuesta al carácter multicultural que caracteriza a la sociedad contemporánea. Al 
tiempo, se argumenta que el roce entre los insights de la teoría curricular crítica y las contribuciones de la teoría social y cultural contemporánea puede favorecer el avance de la discusión de esas cuestiones.

Por otra parte, Bolívar (2004) entiende la multiculturalidad como la existencia de múltiples culturas y la designa además como una situación de hecho, como la diversidad o el pluralismo cultural. Por el contrario, el multiculturalismo, entendido como convivencia de culturas diferentes en igualdad de derechos no designa, una condición, sino una ideología y una orientación, aquella que reclama una política que reconozca las diferencias identitarias. Así, desde el punto de vista del origen moderno de la ciudadanía, establecer un espacio público común (objetivo de la educación pública), una "ciudadanía integradora", con el grave riesgo de ser homogeneizadora o asimiladora, no debe estar basado en la identidad cultural. Bajo dicha homogeneización, la escuela ha excluido la identidad propia de pueblos y culturas. Aquí se propone reformular la concepción de ciudadanía como hecho más complejo.

\section{Debates epistemológicos, enseñanza de las ciencias y diversidad cultural}

Una vez establecidos aspectos generales de la polisemia de la diversidad cultural, nos corresponde abordarla en relación con la enseñanza de las ciencias, debates que hacen énfasis en aspectos epistemológicos. Para Hodson (1993b) existen varios significados sobre el multiculturalismo y la enseñanza de las ciencias, en algunos casos se asocia 1. Un conjunto de estrategias para enfrentar la diversidad étnica y cultural del aula, o 2. Propuestas curriculares para aumentar la autoestima de grupos minoritarios que usualmente han sido excluidos o alienados por la ciencia, o 3 . Una forma de crear conciencia sobre el racismo (y otras formas de discriminación, exclusión y opresión) en la ciencia o la enseñanza de las ciencias.

Para algunos, le concierne a la educación multicultural arreglárselas con los problemas creados con la diversidad cultural y significa un reto para superar las amenazas a niños de minorías étnicas, culturales y religiosas; sin embargo, el fenómeno multicultural en el aula debe entenderse como una oportunidad creada, en un grupo de estudiantes, por la existencia de perspectivas diversas, culturales, étnicas y religiosas. Para el caso de esta propuesta de investigación, interesa profundizar en las perspectivas, acerca de la búsqueda de un fundamento para una educación científica culturalmente sensible a la diversidad, y una caracterización de las relaciones entre conocimiento científico y conocimiento escolar.

Con respecto a la búsqueda de un fundamento para una educación científica multicultural, anotan El-Hani y Sepúlveda (2006) anotan como el debate quedó abierto en los siguientes términos: mientras que Hodson (1993a) se preocupa por una educación científica sensible al contexto cultural, que busca no violentar las creencias que no comparten la visión de mundo de la estructura conceptual de 
la ciencia, Williams (1994), por el contrario, reaccionó criticando la propuesta de Hodson, considerando que, con su defensa de una educación científica sensible a las creencias y experiencias culturales y étnicas de los estudiantes, estaría Hodson realmente violentando el conocimiento científico que, siendo universal, no podría ser entendido en términos multiculturales (El-Hani et al., 2006, p. 165). El debate anterior ha motivado no solo posicionamientos políticos y morales, sino también profundas discusiones filosóficas acerca del estatuto epistemológico de la ciencia moderna occidental y las relaciones con otras formas de conocimiento y su demarcación. Sintéticamente, veamos las posturas emergentes:

1. Los universalistas (Matthews, 1994; Williams, 1994; Siegel, 1997; Southerland, 2000) defienden que la ciencia posee, en cuanto cuerpo de conocimientos y actividad, un carácter universal y no puede ser enseñada en términos multiculturales; Matthews sostiene que el universalismo se basa en que la realidad es la que da la última palabra y que esta particularidad, de ser árbitro final de las adecuaciones de sus enunciados, le concede a la ciencia moderna occidental un poder epistémico superior a otras formas de conocimiento (Matthews, 1994; Siegel, 1997).

2. Los multiculturalistas (Ogawa, 1995; Pomeroy, 1992; Stanley y Brickhouse, 1994, 2001; Snively y Corsilia; 2001) argumentan que el universalismo, y la política de exclusión que se fundamenta en él, es incorrecto desde el punto de vista epistemológico, moral y político y proponen la inclusión de los TEK (Traditional, Ecological, Knowledge) en el currículo de ciencias.

3. Los pluralistas epistemológicos (Cobern y Loving, 2001; López, 1997; ElHani y Bizzo, 1999, 2002; Mortimer, 2000; El-Hani y Mortiner, 2007) defiende, con diferentes argumentos, que el conocimiento científico es una forma específica de conocimiento; sin embargo, no aceptan la discriminación de otras formas de conocimiento y la sobrevaloración de conocimiento científico en detrimento de otros conocimientos. Cobern et al. (2001) argumentan que no se trata de dar más importancia a la ciencia moderna occidental, sino de pensar en la utilidad de la demarcación de formas de conocimiento distintos; la devaluación de otras formas de conocimiento, como los conocimientos ecológicos, el arte, la literatura o la religión, no se debe solamente a la definición tradicional de conocimiento científico, sino al marcado cientificismo existente en nuestras sociedades.

La anterior discusión plantea la necesidad de una caracterización de las relaciones entre conocimiento científico y conocimiento escolar; Molina (2004) realiza una descripción de las relaciones asimétricas que se establecen entre conocimiento científico y conocimiento escolar, originadas en una sobrevaloración del conocimiento científico. En este sentido, cabe señalar que el conocimiento escolar no es equivalente al conocimiento científico para todos los profesores, como indican Martínez y Rivero (2001).

Un enfoque multicultural entiende que los diferentes conocimientos se ponen en contacto cuando se realiza el proceso de enseñanza aprendizaje; Cobern (1994, 1996), Cobern y Aikenhead (1998), Aikenhead (1996, 2001), Costa (1995), Molina 
(2000 y 2005a) han mostrado que el cruce de fronteras culturales es un fenómeno que ocurre continuamente en cualquier aula de clase, incluso en sociedades fundamentalmente urbanas; ellos han apuntado que los alumnos que frecuentan aulas de ciencias en contextos occidentales urbanos participan de diferentes subgrupos culturales.

Como destacan Cobern et al. (1998), los subgrupos encontrados en una determinada cultura pueden ser identificados por la raza, la etnia, el lenguaje, el género, la clase social, la clase de trabajador, la religión, etc., constituyendo subculturas que pueden ejercer una poderosa influencia en el aprendizaje de las ciencias. La propia ciencia escolar puede ser entendida como una subcultura, la cual, en la concepción de Aikenhead (1996), transmite una visión estereotipada de las ciencias, de carácter positivista, alienada de los aspectos sociales, autoritaria y absolutista. En los términos anteriores, los alumnos son frecuentemente impelidos a negociar cruces entre fronteras culturales, sin saber qué están negociando o si lo quieren hacer; a pesar de no recibir una educación para ello, sus fracasos escolares no son comprendidos adecuadamente.

Esta caracterización ha motivado investigaciones que buscan establecer la existencia de barreras culturales en el aprendizaje de las ciencias. (Ogawa, 1989, citado en Cobern,1993; Kawasaki, 1990, 1996). Estos estudios se fundamentan en una visión de mundo distinta de la visióndel mundo que propone la ciencia occidental, encontraron incompatibilidades entre las nociones de causalidad, además de obstáculos en la comprensión de los modelos explicativos, que están asociadas a una serie de discrepancias entre la imagen mecanicista de la naturaleza de la ciencia escolar, los presupuestos materialistas y naturalistas del discurso científico y la imagen de naturaleza presentadas por los alumnos (Cobern, 2000; Cobern et al., 2001).

En el caso de Molina (2000), Molina, López y Mojica (2005) y Molina (2007), las relaciones entre diferentes sistemas de conocimiento se entienden como intercambios que tienen sus orígenes en la conformación de las mismas culturas; se encontró, en un grupo de niños urbanos, que existen diferentes visiones en torno a la adaptación vegetal, en las cuales se identificaron perspectivas occidentales y no occidentales. Por otra parte, las ideas de naturaleza de niños de cuatro grupos distintos (citadinos, inmigrantes campesinos, campesinos de influencia U'wa) evidencian intercambios, diferencias y criterios compartidos, cuyos sus orígenes pueden remitirse a los siglos XVIII, XIX y XX de la historia nacional.

\section{Concepciones de los profesores de ciencias}

Otro campo de la enseñanza de las ciencias que registra la inclusión de lo cultural, específicamente hacia el reconocimiento de la diversidad cultural, es el de las concepciones de los profesores. De acuerdo con el anterior propósito, el material estudiado se organizó en tres tendencias: 1. Concepciones epistemológicas de los 
profesores, 2. Concepciones de ciencia de los profesores y enseñanza 3. Aperturas hacia una alternativa más contextual. La organización de los antecedentes permite visualizar una tendencia que varía, de un carácter más universalista, hacia visiones más contextuales. La revisión también contempla los avances metodológicos de las investigaciones en torno a las concepciones de los profesores.

\section{Concepciones epistemológicas de los profesores}

Con el objetivo de ubicar el trabajo de investigación en la línea de las concepciones de los profesores, nos apoyamos en los de trabajos de Harres (1999), Porlán, Rivero y Martín (2000), Brown, Luft, Roehrig y Kern (2006). Porlán, Rivero y Martín (2000) señalan resultados de diferentes investigadores como Gordon (1984), Lederman (1992), Martín (1994), Porlán (1996), Porlán y Martín (1996), Porlán et al. (2000), entre otros, desde los cuales resaltan que la visión absolutista, de los planteamientos empirista y racionalista, de la ciencia constituye el obstáculo central para el desarrollo de una epistemología constructivista y, en particular, que impide considerar al conocimiento escolar (y el propio conocimiento profesional) como un conocimiento epistemologicamente diferenciado y no como una reproducción enciclopédica, fragmentada y simplificada de las disciplinas y el conocimiento de los alumnos como un conocimiento alternativo (Porlán et al., 1996, p. 520).

Las investigaciones a nivel nacional también a evidenciar este problema, pues indcan que prevalece una visión empirista en los profesores (Barajas, 2006), además de identificar posiciones eclécticas (Gallego y Pérez, 1999) y caracterizar paradigmas epistemológicos diferentes (Reyes, Salcedo y Perafán, 2001). Cabe destacar que diferentes investigaciones han coincidido en señalar el carácter no uniforme de estas concepciones, tal como lo indican Koulaidis y Ogborn (1989) y Porlán (1989). Hoy se contempla la necesidad de tener en cuenta la amplitud de las posiciones epistemológicas para dar razón de las creencias de los profesores (Martínez, 2000; Perafán, 2004; Mosquera y Furió, 2008).

En el caso de Harres (1999), quién realizó una revisión sobre las CNC (Concepciones sobre la Naturaleza de la Ciencia) apoyado en los trabajos de Lederman (1992), establece que las investigaciones sobre CNC de los profesores y su incidencia en sus prácticas de enseñanza son las más recientes. Igualmente, apoyado en otras revisiones (Ledermann, 1992; Koulaidis y Ogborn, 1995; Porlán y Rivero 1998), señala que, teniendo en cuenta sus resultados, presupuestos epistemológicos y metodológicos, las investigaciones realizadas entre los años sesenta y ochenta se desarrollaron independientemente del contexto cultural, de la experiencia docente y de su contexto de actuación; se asume que las CNC de los profesores son empírico-inductivistas.

Se puede comprender esta conclusión en tres situaciones: 1 . En cuanto a los objetivos de las investigaciones que buscaban establecer el grado de adecuación 
de las concepciones de los profesores, a los de la filosofía y epistemología. 2. Los presupuestos filosóficos y epistemológicos adoptados se centran, por una parte, en visiones racionalistas que no consideraban aspectos contextuales y más relativistas de la ciencia y, por otra, no se tienen en cuenta las diferencias entre las tesis de los filósofos de la ciencia, por el contrario, parece asumirse que entre los filósofos de las ciencias ya no hay debates y que el consenso entre ellos está definitivamente establecido y generalizado. 3. La elaboración de categorías previas a la recolección del material determina los resultados obtenidos (CNC empiristas, inductivistas), sin reconocer la existencia de modelos que están en conflicto con los de la ciencia (Lucas, 1975). En esta dirección, Koulaidis et al. (1989) defienden que, cuando se incluye en el instrumento de recolección de datos sobre visiones de la ciencia y se investigan las $\mathrm{CNC}$ como un todo, se puede constatar una posición más contextualizada y un papel menos relevante de la racionalidad en las concepciones de los profesores; Guilbert y Meloche (1993) encontraron tanto visiones relativistas como absolutistas; Ogborn et al. (1989) también constataron una visión más flexible en relación con las CNC de los profesores.

Un trabajo más reciente (Brown, Luft, Roehrig y Kern, 2006) ratifica lo anterior; dichos investigadores, a partir de una revisión de diferentes perspectivas filosóficas de la ciencia, amplían el rango de caracterización de las concepciones epistemológicas, clasificándolas en cuatro grandes grupos de acuerdo con una compresión de CNC centrada en productos, procesos, paradigmas y como un fenómeno situado; el ajuste del instrumento en una prueba piloto (realizada con profesores de ciencias de nivel básico) indujo a la fusión de la perspectiva paradigmática y situada; de esta forma, las CNC fueron afectadas por la sociedad y la política. Con respecto a este último aspecto, los profesores experimentados se ubican en la perspectiva que hace énfasis en las diferentes respuestas. En cuanto a este último resultado, muestra cómo, desde una perspectiva epistemológica, pero con enfoques más externalistas, las CNC de los profesores tienden a relativizarse.

\section{Concepciones de ciencia de los profesores y enseñanza}

La revisión realizada por Porlán et al. (2000) se refiere a las concepciones didácticas de los profesores de ciencias, entre las que se incluyen investigaciones que se caracterizan, a nivel metodológico, por una división en tres enfoques investigativos. El enfoque cientificista pretende la generalización de resultados, muestras grandes, uso de cuestionarios, enfoques cuantitativos (p. ej. Victor, 1997; Bauch 1984). El enfoque interpretativo busca dar razón de las creencias, muestras reducidas, metodologías cualitativas (p. ej. Elbaz, 1981; Buitnik y Kemme, 1986; Oberg, 1986). El enfoque crítico que está centrado en la investigación para la transformación de las prácticas de los profesores, que integran enfoques cualitativos y cuantitativos (p. ej. Marrero, 1994; Porlán et al., 2000). 
En esta perspectiva incluimos los trabajos de Utges y Pacca (2003) y Rodríguez y Marrero (1994), quienes serán la referencia para nuestra investigación. Finalmente, la revisión de lo antes señalado destaca que las investigaciones revisadas muestran una mayor tendencia a concebir la enseñanza como una actividad centrada en la explicación del profesor, con los contenidos como eje director de la dinámica de la clase y controlada y dirigida por este (p. 513). En cuanto a la relación entre CNC y enseñanza, Porlán y Rivero (1998) encuentran que son complejas y relativas; así, el conocimiento de los profesores puede estar compuesto de múltiples relaciones: personalidad, experiencias previas, condiciones contextuales, valores, etc. que influyen en su conducta.

Sin embargo, las relaciones entre las concepciones de los profesores, sobre el conocimiento científico y las propuestas de enseñanza, son variadas según las perspectivas de diferentes autores. Como señala Martínez (2000), por ejemplo, es posible identificar investigaciones que señalan como las concepciones no son causa de una determinada práctica docente; tal es el caso de Hodson (1993a), citado por Fernández (2000) y Prawat (1992), para quienes no siempre es posible considerar la influencia de las concepciones científicas en las concepciones sobre la enseñanza y el aprendizaje.

Por otra parte, son numerosas las investigaciones que muestran que hay una relación estrecha entre estas concepciones de los profesores y sus propuestas de enseñanza. Por ejemplo Tobin y Macrobbie (1997), Fernández (2000), Smith (2000), Porlán et al. (2000) señalan que las concepciones sobre el conocimiento científico de los profesores están asociadas con sus prácticas. Además, es posible identificar diferentes formas de asumir dichas relaciones (Porlán, 1989; Martínez, 2000; Perafán, 2004), pues se reportan datos contradictorios que señalan su complejidad y la incidencia de diferentes factores, por ejemplo, el aspecto analizado (ontológico, la validez, el contenido a ser enseñado) o la experiencia docente.

\section{Aperturas hacia una alternativa más contextual}

Con respecto a las investigaciones que exploran relaciones entre las concepciones de los profesores sobre la ciencia y la enseñanza, teniendo en cuenta aspectos más contextuales, se pueden ubicar esencialmente dos tendencias: la perspectiva situada y la cultural, las cuales se constituyen en aportes relevantes para nuestra investigación. Permiten importantes desarrollos conceptuales al mostrar que tanto las concepciones de ciencia (en diferentes tradiciones académicas) como las de los profesores sobre su trabajo, están fuertemente determinadas por los contextos políticos, sociales, históricos y culturales.

En el caso de la perspectiva que hemos denominado situada, encontramos a Barnet y Hodson (2001), quienes ubican al profesor en una sociedad, en un grupo social identificable, o subgrupo, que posee sus patrones distintivos en ella y que es socialmente validado, con creencias, expectativas y valores que determinan o definen la 
manera en que sus miembros actúan, juzgan, toman decisiones, resuelven problemas $\mathrm{y}$ asumen determinados enfoques. Para estas posturas, las creencias y valores que conforman los conocimientos del profesor se manifiestan en la práctica y en las decisiones tomadas; los profesores de ciencias viven en múltiples interacciones de micromundos, cada uno de ellos anidado en el más amplio mundo social de la educación.

En esta perspectiva situada, encontramos a Wells (1998), quien a partir de un estudio de caso permite formular una visión o concepción de la enseñanza basada en el enfoque sociocultural, en la cual la actividad, el desarrollo del discurso, las prácticas y mediaciones utilizadas, determinan los contenidos tratados. Wells, al reconocer el contexto (apuntado por Barnett et al. 2001 y según Molina, 2000), permite mostrar una de sus características, en la medida en que, tanto los profesores como los alumnos, forman una comunidad (la clase) que es única.

El pasado tanto de los estudiantes como de los profesores, sus creencias, valores, dificultades y potencialidades se constituyen en referencias. Los estudiantes tienen sus propios intereses, fortalezas y limitaciones individuales, así como sus propias experiencias pasadas, personales y culturales. Igualmente, cada maestro tiene un estilo particular de la enseñanza que se basa en creencias personales, valores y experiencias pasadas.

Como Scribner (1984, p. 39, citado en Barnet et al., 2001) dice, “el pensamiento [...] experto, práctico es metadirigido, adaptable y varía con el cambio de las propiedades de los problemas y la evolución de las condiciones en la tarea, con el medio ambiente". Según Claxton (1987), todos creamos miniteorías sobre el mundo que nos rodea. En síntesis, el profesor está situado en el salón de clase, en las minucias de la vida cotidiana (contextos educativos y micro mundos). Lo anterior se ha denominado conocimiento pedagógico contextual. Las fuentes de este conocimiento son internas y externas.

En este sentido, Clandinin y Connelly (1995), citados en Barnet et al. (2001), se refieren al conocimiento pedagógico con la metáfora del paisaje, se trata de un conocimiento que implica saber algo acerca de dos componentes, de dos paisajes: el de la sociedad del conocimiento, es decir, todos los conocimientos pertinentes para el eficaz funcionamiento de la sociedad, y en segundo lugar, el conocimiento del paisaje educativo, es decir, todos los conocimientos relativos a los asuntos educativos.

Con respecto a la tendencia cultural, Aikenhead y Huntley (2000) realizaron una investigación de las visiones de los profesores sobre la ciencia occidental escolar enseñada a estudiantes aborígenes. Los profesores generalmente veían la ciencia occidental como el contenido del curso o como un modo de explorar la naturaleza, no como una cultura extranjera, como es experimentada por muchos de sus estudiantes aborígenes. El conocimiento aborigen fue respetado por los profesores de ciencias, pero sólo una cantidad mínima fue incorporada a la clase sin integrarlo a la ciencia escolar.

Los profesores pensaron que el acto de aprender ciencia no se relacionaba con las cosmovisiones aborígenes de sus estudiantes. El desinterés por cursar carreras de ciencias no fue abordado por los entrevistados y aquellos que brindaron explicación 
la realizaron a partir de la existencia de un déficit en los estudiantes; pocos profesores pensaron que tal vez este desinterés está relacionado con los enfoques de su plan de estudios, o con la manera en que ellos desarrollan su enseñanza. En este mismo sentido, la investigación de Smolen, Colville-Hall, Liang y MacDonald (2006) muestra que no existe una correlación entre el discurso declarativo, que reconoce la diversidad cultural, y la práctica de enseñanza desarrollada por los docentes.

En la investigación de Sawyer (2000), cuando se preguntó a los profesores sobre la necesidad de adaptar el currículo a la diversidad cultural (los valores culturales) de los estudiantes, si bien la mayoría de ellos se manifestó en favor de la adaptación curricular, el compromiso con estas creencias resultó incompatible. Los docentes prefirieron discutir la adaptación curricular en relación con las características individuales del estudiante, pero no estuvieron dispuestos a construir planes de estudio para los estudiantes considerando la diversidad de valores culturales.

Por otra parte, Rudolph (2003) realiza un análisis de las influencias de los contextos históricos en la diversidad de prácticas científicas; estas perspectivas incluyen nuevos aspectos que conforman visiones más amplias de la ciencia -que involucran varios sistemas de conocimientos indígenas u otros no occidentales del mundo- que de hecho están motivadas por un deseo de alterar el panorama político de la educación científica (por ejemplo, Hodson, 1999; Kawagley, Norris-Tull, y Norris-Tull, 1998; Snively et al., 2001; Stanley y Brickhouse, 2001).

Para Rudolph, se requiere de una mayor conciencia de las consecuencias sociales y políticas implícitas en los diferentes puntos de vista sobre la ciencia, que encarnan una discusión más abierta, de los objetivos sociales de la enseñanza de la ciencia, que debe servir cuando todo está dicho y hecho. En este sentido, encontramos los trabajos de Cobern et al. (2001), quienes realizan una reflexión acerca del origen multicultural de la ciencia.

Estos actores examinan su definición, respecto de las perspectivas multiculturales, considerándo las tesis de distintos filósofos de la ciencia; frente a las perspectivas universalistas, es decir, la visión estándar, anotan que los cuestionamientos sobre universalidad no se dan alrededor de los fenómenos mismos, sino en torno a la manera en que se plantean las proposiciones para considerar cada fenómeno en sí, se dan respecto de la manera en que empleamos el discurso para comunicarnos frente a los fenómenos, y en cuanto a los valores que añadimos a dichos fenómenos, así como a las varias maneras que tenemos de entender y comprenderlos, incluyendo la manera estándar que ofrece la ciencia.

\section{A modo de conclusión}

El desarrollo de la investigación y la innovación en educación en ciencias ha sido vertiginoso, especialmente en las últimas tres décadas. En este contexto, la investigación sobre las concepciones y prácticas de los profesores de ciencias ha resultado particularmente relevante, si se tiene en cuenta que son ellos quienes pueden, de 
manera decidida y explícita, incorporar o no los resultados de los progresos en este campo del conocimiento educativo.

Como se ha anotado en este artículo, son variadas las investigaciones que han abordado esta situación. Sin embargo, son pocos los estudios en torno a las concepciones que los profesores de ciencias desarrollan sobre diversidad cultural y sus incidencias en el trabajo docente. Quizás la poca comprensión de los profesores, en torno a la diversidad cultural en el aula, puede desempeñar un papel de suma importancia en los procesos de aprendizaje de las ciencias y en las valoraciones que los estudiantes conceden a la noción de conocimiento científico.

De igual forma, diversos estudios sobre las concepciones alternativas de los estudiantes, esquemas o ideas previas, se han identificado con concepciones y prácticas aceptadas como válidas en el contexto de la ciencia occidental; sin embargo, poco se han estudiado en su relación con su naturaleza cultural. Todo esto cobra mucho más sentido cuando deseamos desarrollar esta investigación en un país como Colombia, caracterizado por su altísima diversidad cultural y por sus condiciones pluriétnicas.

Finalmente, queremos reiterar que, a partir de los trabajos referenciados sobre las concepciones de los profesores de ciencias naturales (epistemológicas, sobre la enseñanza), existe la necesidad de realizar un descentramiento de lo meramente epistemológico y didáctico, hacia perspectivas más contextuales, y así reconocer que éstas son un campo complejo y problemático de investigación aún no resuelto.

Por ello, hemos abordado en la investigación preguntas orientadoras como: ¿cuáles son las concepciones de los profesores de ciencias sobre la diversidad cultural?, ¿es reconocida esta diversidad?, ¿qué tan sensibles son ellos a esta diversidad respecto a sus propuestas de enseñanza? ¿perciben los profesores la necesidad de modificar los objetivos, contenidos y estrategias de enseñanza de las ciencias atendiendo a esa diversidad cultural? Esperamos dar cuenta, muy pronto, de los avances respecto a estas preguntas. 


\section{Referencias bibliográficas}

Aikenhead, G. (1996) Science education: Border crossing into the subculture of science. Studies in Science Education, 27, 1-52.

Aikenhead, G. (2001). Students' ease in crossing cultural borders into school science. Science Education, 85, 180188.

Aikenhead G. \& Huntley, B. (2000). Teachers' Views on Aboriginal Students Learning Western and Aboriginal Science. Obtenido el 20 de agosto de 2008, desde http://www.usask.ca/education/ people/aikenhead/cjne.pdf

BARAJAS, D. (2005). Las relaciones que establece el profesor universitario de biología con la disciplina que enseña. Una explicación epistemológica en dos estudios de caso en la Universidad Tecnológica del Chocó. Tesis de Maestría Facultad de Educación, Universidad de Antioquia.

Barnett, J \& Hodson, D. (2001). Pedagogical Context Knowledge: Toward a Fuller Understanding of What Good Science Teachers Know. Science Education 85, 426-453.

Bauch, P. (1984). The impact of teachers' instructional beliefs on their teaching: implications for research and practice. Reunión Anual de la AERA. (en versalita minúsculas AERA). New Orleans: Abril
Boaventura De Souza Santos. (1989). Intoduçao a Uma Ciência Pós Moderna. Rio de Janeiro: Graal

Bolivar, A. (2004). Ciudadanía y escuela publica en el contexto de la diversidad cultural. Revista Mexicana de Investigación Educativa, 9(20), 15-38.

Brown, M., Luft, L., Roehrig, G. \& Kern, A. (2006). Beginning Science Teachers' Perspectives on the nature of Science: The Development of a Nature of Science Rubric. Presented at The ASTE 2006. International Conference, Portland, Oregon. January 12, 2006.

Bruner, J. (1990). Actos de significado: Más allá de la revolución cognitiva. Madrid: Alianza.

Buitink, J., Kemme, S. (1986) Changes in students-teacher thinking. European Journal of Teacher Education, 9 (1), 75-84.

Candau, V. M, \& Nehme Simão e Koff, A. M. (2006). Conversas com... sobre a didática e a perspectiva multi/ intercultural. Educacao \&o. Sociedade, 27(95), 471-493.

Clandinin, D. J., \& Connelly, F. M. (1995). Teachers' professional knowledge landscapes. New York: Teachers College Press.

Claxton, G. (1987). Vivir y aprender. Madrid: Alianza Psicología. 
Claxton, G. (1994). Educar mentes curiosas: el reto de la ciencia en la escuela. Madrid: Visor.

Cobern, W. (1991). World View Theory and Science Education Research. Manhattan- Kansas: NARST.

Cobern, W. (1994). Point: Belief, understanding, and the teaching of evolution. Journal of Research Science Teaching 31, 583-590.

Cobern, W. (1996). Worldview theory and conceptual change in science education. In Science Education, 80(5), 579-610.

Cobern, W. \& Aikenhead, G. S. (1998). Cultural aspects of learning science. In Fraser, B. J. \& Tobin, K. (Eds.), International Handbook of Science Education (pp. 39-52). Dordrecht: Kluwer Academic Publishers:.

Cobern, W. \& Loving, C. (2001). Defining "Science" in a Multicultural World: Implications for Science Education. In Science Education, 85, 50-67.

Costa, V. B. (1995). When science is "another world": Relationships between worlds of family, friends, school, and science. Science Education, 79(3), 313-333.

D’Ambrosio, U. (1998). Etnomatemática. São Paulo: Editora Ática.

Douglas, M. (1998). La elección entre lo somático y lo espiritual: algunas preferencias médicas. Estilos de pensar. (pp.38-64), Barcelona, Gedisa.
Elbaz, F. (1981) The teachers' practical knowledge: report of a case study. Curriculum Inquiri, 11 (1), 43-71.

El-Hani, C. N., \& Bizzo, N. (2002). Formas de construtivismo: Mudanc a conceitual e construtivismo contextual. Ensaio: Pesquisa em Educação em Ciencias, 4, 1-25.

El-Hani, C. N., y Sepulveda, C. (2006). Referenciais teóricos y subsídios metodológicos para a pesquisa sobre as relações entre educação científica e cultura. Teixeira Dos Santos, F \& Greca, M. A. Pesquisa em ensino de ciências no Brasil e suas metodologias. (pp 161-212), Rio Grande do Sul: Unijuí Editora.

El-Hani, C. N. \& Mortimer, E. (2007). Multicultural education, pragmatism, and the goals of science teaching. Cultural Studies of Science Education 2, 657-702.

Escobar, A. (2003). Mundos y conocimientos de otro modo. Tabula Rasa 1, 51-86.

Fernández, E. (2000). Análisis de las concepciones docentes sobre la actividad cientifica: una propuesta de transformación. Tesis Doctoral, para la obtención del título de Doctor en Educación. España: Universitat de Valéncia.

Ferreira Pires J. (1995). Cultura é memória. Revista UPS, 24, 114-120.

FORQUIN J-CL. (1993). Escola e cultura. As bases sociais e epistemológicas do conhecimento escolar. Porto Alegre: Ed. Artes. 
García, Canclini, N. (2004). Diferentes, desiguales y desconectados. Mapas de la Interculturalidad. Buenos Aires: Gedisa.

Gallego, R. y Pérez, R. (1999). El problema del cambio en las concepciones epistemológicas, pedagógicas y didácticas. Bogotá: Universidad Pedagógica Nacional.

Geertz, C. (1996). Los usos de la diversidad. Barcelona, Paidós.

Guilbert L. \& Meloche, D. (1993). L'idée de science chez des enseignants en formation: un lien entre L'histoire des science et L'heterogénéité de visions? Didaskalia, 2, 7-30.

Giroux, H. (1997). Cruzando límites. España: Ed Gedisa.

Gomes, N. L. (2003). Cultura negra e educação. Revista Brasilera. Educação, 23, $75-85$.

Gordon, D. (1984). The Image of Science, Technological Consciousness and Hidden Curriculum. Curriculum Inquiry, 14 (4), 367-400.

Guillen, F. (1996) El poder Político en Colombia. Santa fe de Bogotá: Planeta Colombiana Editorial.

Gutiérrez, C. B. (1997). Reflexiones Hermenéuticas en Torno de "Ética y diversidad cultural". Ética y diversidad cultural Bogota: Fondo de Cultura Económica, 71-84.

HARres, J.B.S. (1999) Uma revisão de pesquisas nas concepções de professores sobre a natureza da ciência e suas implicações para o ensino. Investigaciones en Enseñanza de las Ciencias, 4(3), 197-211.

Hederich, C. y Camargo, A. (1999). Estilos cognitivos: Resultados en Cinco Regiones Culturales. Universidad Pedagógica Nacional, Centro de Investigaciones Santa fe de Bogotá D.C., Colombia: (CIUP), COLCIENCIAS.

Hodson, D. (1993a). In search of a rationale for multicultural science education. Science Education, 77, 685-711.

Hodson, D. (1993b). Philosophic stance of secondary school science teachers, currículo experiences and children's understanding of science: some preliminary findings. Interchange, 24 (1-2), 41-52.

Hodson, D. (1999). Going Beyond Cultural Pluralism: Science Education for Sociopolitical Action. Science Education 83, 775-796.

Kawagley, A. O., Norril-Tull, D. \& Norris-Tull, R. A. (1998). The indigenous worldview of Yupiang culture: Its scientific nature and relevance to the practice and teaching of science. Journal of Research in Science Teaching, 35(2), 133-144.

Koulaidis, V. \& Ogborn, J. (1989). Philosophy of science. An empirical study of teacher's views. International Journal of Science Education, 11 (2), 173-184.

Koulaidis, V. \& Ogborn, J. (1995). Science teachers's philosophical assumptions: how well do we understand 
them?. International Journal of Science Education, 17(3), 273-283.

LANGon, M. (Para un modelo dialógico del pensar: Reflexiones a partir de un espacio de diálogo Intercultural. Tomado de la Conferencia presentada en el II Congreso de Filosofía Intercultural, São Leopoldo, R.S., Brasil 9 de abril de 1997. Obtenido desde (bttp://fp.chasque.net/ relacion/ anteriores/9707/reflexiones. $\mathrm{htm}$ )

Lederman, N. (1992). Students' and Teachers' Conceptions of Nature of Science: A Review of the Research. Journal of Research in Science Teaching, 29 (4), 331-359.

López, P. (2000). Una oportunidad para aprender. La formación intercultural en la formación del profesorado, España: Cabildo de Tenerife, Área de desarrollo económico.

Lloyd, C. (1995). As Estruturas da História. Rio de Janeiro: Jorge Zahar Editor.

Matthews, M. R. (1994). Science Teaching: The Role of History and Philosophy of Science. New York: Routledge.

Martín, R. (1994). El conocimiento del cambio químico en la formación inicial del profesorado. Estudio de las concepciones disciplinares y didácticas de los estudiantes de magisterio. Tesis Doctoral, Facultad de Educación, Universidad de Sevilla, España.

Martínez, C. (2000). Las propuestas curriculares sobre el conocimiento escolar en el área de conocimiento del medio: dos estudios de caso en profesores de primaria. Tesis Doctoral. España: Universidad de Sevilla.

Martínez y Rivero (2001). El conocimiento profesional sobre el conocimiento escolar en la clase de conocimiento del Medio. Revista Investigación en la Escuela, 45, 65-75.

Marrero, J. (1994) Las teorías implícitas del profesorado, vínculo entre la cultura y la práctica de la enseñanza. En Rodrigo, M. J., Rodríguez, A. \& Marrero, J. (Eds), Las Teorías Implícitas. Una Aproximación al Pensamiento Cotidiano. Ed. Aprendizaje Visor: Madrid.

Mauss, M. (1974). As técnicas corporais. Sociologia e antropologia. São Paulo: EPU. pp, 209-233.

Molina, A. (2000). Conbecimento, Cultura e Escola: Um estudo de suas Inter-relações a partir das idéias dos alunos (8-12 anos) sobre os espinhos dos cactos. Tesis doutoral, Faculdade de Educacao, Universidade de São Paulo, Brasil.

Molina, A. (2002): Conglomerado de relevancias de niños, niñas y jóvenes. Revista Científica, 4(1), 187-200.

Molina, A. (2004). Enfoques culturales en investigaciones acerca de la enseñanza, el aprendizaje, los textos escolares en la evolución de la vida. Cuadernos de Investigación $N^{\circ}$ 4, 9-33.

Molina, A., López, D. \& Mojica, L. (2005). Ideas de los niños y niñas sobre la naturaleza: un estudio comparado. Revista Científica, 7(1), 41-62. 
Molina, A. (2005a). El "otro" en la constitución de identidades culturales. En Piedrahita, C. \& Paredes, E. (Eds.), Cultura política, identidades y nueva ciudadanía, vol. 2 (pp.139-169). Cúcuta: Sic Editorial.

Moreira, F, B. (2002) Currículo, diferencia cultural e diálogo. Educação \& Sociedade, 23(79), 15-30.

Mortimer, E. F. (1998). Sobre chamas e cristais: a linguagem cotidiana, a linguagem científica e o Ensino de Ciências. In: Chassot, A. \& Oliveira, R. J. Ciência, ética e cultura na educação (99-118). São Leopoldo: UNISINOS.

Mosquera, C. J. y Furió, C. J. (2008). El cambio didáctico en profesores universitarios de química a través de un programa de actividades basado en la enseñanza por investigación orientada. Revista Didáctica de las Ciencias Experimentales y Sociales, 22, 115-154.

Ogberg, A. (1986) Using construct theory as a basis for research into teacher professional development. Journal of Curriculum Studies, 19 (1), 55-65.

Ogawa, M. (1995) Science education in a multi-science perspective. Science Education 79, 583-593.

Perafán (2004). La epistemología del profesor sobre su propio conocimiento profesional. Bogotá: Universidad Pedagógica Nacional, Colección Tesis Doctorales 1.

Pomeroy, D. (1992). Science across cultures: building bridges between traditional Western and Alaskan native cultures. In Hills, S. (Ed.), History and philosophy of science education, vol. 2 (pp. 257-268). Kingston Ontario: Quenn's University.

Porlán, R. (1989). Teoría del conocimiento, teoría de la enseñanza y desarrollo profesional. Las concepciones epistemológicas de los profesores. Tesis Doctoral Facultad de Educación, Universidad de Sevilla, España.

Porlán, R. (1996). Proyecto Docente. Universidad de Sevilla. Documento por publicar. Departamento de Didáctica de las Ciencias.

Porlán, R. \& Martin, R. (1996), Ciencia, profesores y enseñanza: unas relaciones complejas. Alambique, 8 , 23-32.

Porlán, R. \& Rivero, A. (1998). El conocimiento de los profesores. Sevilla: Díada Editora.

Porlán, R., Rivero, A. y Martín, R. (2000). El conocimiento del profesorado sobre la ciencia, su enseñanza y aprendizaje. En Perales, F. y Cañal, P. (Comps.), Didáctica de las Ciencias Experimentales. Alcoy: Marfil.

Pozo, J. I., (2003). Adquisición de Conocimiento. Madrid: Ediciones Morata, S.L.

Pozo, I. I., Gómez Crespo, M.A. (1998) Enseñar y aprender ciencias. Madrid: Ediciones Morata, S.L.

Prawat, R. (1992). Teachers' belief about teaching and learning: a constructivist perspective. American Journal of Education, 100 (3), 354-395. 
Reyes. L., Salcedo L. E. y Perafán, A. (2001). Acciones y creencias. Análisis e interpretación de creencias de docentes en biología y ciencias naturales. Tomo

IV. Bogotá: Universidad Pedagógica Nacional.

Rodrigo, M. J., Rodríguez, A. y MARRERO, J. (1993) Las teorías implícitas. Una aproximación al pensamiento cotidiano. Madrid: Ed. Aprendizaje Visor.

Rudolph, J. (2003). Portraying Epistemology: School Science in Historical Context. Inc. Science Education 87, 64-79.

SAWYer, R. (2000). Adapting Curriculum to Student Diversity: Patterns and Perceptions among Alternate-Route and College-based Teachers. Urban Review, 32(4), 343-63.

SCRIBNER, S. (1984). Studying working intelligence. In B. Rogoff \& J. Lave (Eds.), Everyday cognition: Its development in social context (pp. 9-40). Cambridge, MA: Harvard University Press.

Siegel, H. (1997). Science education: Multicultural and Universal. Interchange 28, 97-108.

Smith, D. (2000). Content and Pedagogical Content Knowledge for elementary Science Teacher Educators: Knowing our Students. Journal of Science Teacher Education, 11 (1), 27-46.

Smolen, L., Colville-Hall, S., Liang, X. \& Mac Donald, S. (2006). An Empirical Study of College of Education Faculty's Perceptions, Beliefs, and Commitment to the Teaching of Diversity in
Teacher Education Programs. at Four Urban Universities [Abstract]. In Urban Review: Issues and Ideas in Public Education, 38(1), 45-61.

Snively, G. \& Corsiglia, J. (2001). Discovering indigenous science: Implications for science education. Science Education, 85, 6-34.

Stanley, W. B. \& Brickhouse, N. W. (1994). Multiculturalism, universalism, and science education. Science Education, 78, 387-398.

Stanley, W. B. (2001). Teaching sciences: The multicultural question revisited. Science Education 85, 35-49.

Southerland, S. (2000). Epistemic Universalism and short comics of curricular multicultural Science Education. Science \& Education 9, 289-307.

SHWEDER R. (1991). La rebelión romántica de la antropología contra el iluminismo, o el pensamiento es más que razón y evidencia. En Geertz C., Clifford J. et al. El surgimiento de la antropología posmoderna. (pp 78-113). México D.F.: Gedisa editorial.

Snively, G. \& Corsiglia, J. (2001). Discovering indigenous science: Implications for science education. Science Education, 85, 6-34.

Stanley, W. B. \& Brickhouse, N. W. (1994). Multiculturalism, universalism, and science education. Science Education, 78, 387-398.

Subercaseux, B. (2005). Diversidad cultural: el otro y el nosotros; En Net, M., Walker, et al. Diversidad cultural: el valor 
de la diferencia, Chile: LOM Ediciones LOM Ediciones; Chile.

Tobin, K. \& Mcrobbie, C. J. (1997). Belief about the nature of Science and the Enacted Science Curriculum. Science \& Education, 6, 355-371.

Toledo, U. (1998). La epistemología según Feyerabend. Cinta de Moebio, 4. Edición Digital.

Toulmin, S. (1977). La comprensión humana. El uso colectivo y la evolución de conceptos. Madrid: Alianza Ed.

Tovar, H. (1992). Colombia: lo diverso, lo múltiple y la magnitud dispersa. Maguare 8(7), 47-81.

TyLer, CH. (1993). El multiculturalismo y la política del reconocimiento. México: Fondo de Cultura Económica.

Utges, G. y Pacca, J. (2003). Análisis factorial en la caracterización de representaciones implícitas. Reflexiones metodológicas a la luz de algunas investigaciones realizadas.

Velho G. (1978). O conceito de Cultura e o Estudo de Sociedades Complexas: uma perspectiva antropológica. Artefato (1) Rio de Janeiro.

Victor, J. (1976). Relation between teacher belief and personality in four samples of teacher trainees. Journal of Experimental Education, 45, 4-9

Wells, G. (1998). Da adivinhação a previsão: discurso progressivo no ensino e na aprendizagem de ciências. Coll, C. \& Edwards. Ensino, aprendizagem e discurso em sala de aula: Aproximações ao discurso educacional, Armed Editora: São Paulo.

Williams, H. (1994). A critique of Hodson's "In search of a rationale for multicultural science education". Science Education, 78, 515-520. 